Cahiers d'études italiennes

9| 2009

NOVECENTO... E DINTORNI

Images littéraires de la société contemporaine (4)

\title{
Sebastiano Vassalli: da abitante del vento a seguace del nulla
}

Hanna Serkowska

\section{(2) OpenEdition}

Journals

Edizione digitale

URL: http://journals.openedition.org/cei/192

DOI: $10.4000 /$ cei.192

ISSN: 2260-779X

Editore

UGA Éditions/Université Grenoble Alpes

\section{Edizione cartacea}

Data di pubblicazione: 15 juillet 2009

Paginazione: 81-90

ISBN: 978-2-84310-145-8

ISSN: 1770-9571

Notizia bibliografica digitale

Hanna Serkowska, «Sebastiano Vassalli: da abitante del vento a seguace del nulla», Cahiers d'études italiennes [Online], 9 | 2009, online dal 15 janvier 2011, consultato il 27 mars 2021. URL: http:// journals.openedition.org/cei/192 ; DOl: https://doi.org/10.4000/cei.192 


\title{
SEBASTIANO VASSALLI: DA ABITANTE DEL VENTO A SEGUACE DEL NULLA
}

\author{
Hanna Serkowska \\ Uniwersytet Warszawski
}

[...] il cielo infinito non [è] deturpato dall'ombra di Nessun Dio.

S. VAsSALli, La notte della cometa.

Per cercare le chiavi del presente, e per capirlo, bisogna uscire dal rumore: andare in fondo alla notte, o in fondo al nulla...

\section{S. VAssalli, La Chimera.}

Tutti quei morti, [...], sono morti per niente, e il resto è merda: la guerra, il seguito, tutto. [...] La storia è merda, Sebastiano. Secolo dopo secolo, tonnellata sopra tonnellata.

Un immenso letamaio e basta...

S. Vassalli, L'oro del mondo.

Vassalli migliore, il Vassalli storico, autore di romanzi storici in cui la vicenda passata diventa una sorta di allegoria del mondo di oggi ${ }^{1}$, uno specchio del presente (diciamo a margine che l'etichetta di uno "storico" a

I. Il legame che unisce il passato al presente è di tipo analogico: l'autore sceglie di ambientare la sua storia nel ‘6oo non perché egli abbia una predilezione per quell'epoca (nel '70o ambienta, infatti, Marco e Mattio e nell'‘80o e '900 Il cigno), ma perché vi ha trovato problemi che lo riconducono all'oggi, che gli permettono di capire e di parlare del giorno di oggi. Vassalli crede insomma nella "contemporaneità della storia" nel senso in cui ne parlava Croce intendendo che lo studio del passato parte sempre dalle idee sul e del giorno di oggi. La storia per lo scrittore è sempre contemporanea, essa è tutt'al più una sorta di "autobiografia del presente", o per citare il titolo del romanzo vassalliano (in cui il " 68 è l'immediato precedente e causa di molti dilemmi di oggi), "archeologia del presente"; aiuta a comprendere il presente che di per se stesso non è in grado di raccontare nulla, perché il presente è ridotto al rumore, è la manopola della televisione. Vassalli rimane infatti 
un certo punto gli è diventata troppo stretta, per cui, nel 1995 egli avrebbe scritto un romanzo futuristico, 30I2, ma anche lì troviamo uno specchio, seppur progettato in avvenire, del presente ${ }^{2}$ ), sembra un erede di Manzoni, cui si riferisce ambientando la trama de La Chimera nel '6oo, ai tempi della Controriforma, fanatismo e intolleranza, roghi e caccia alle streghe. Sembra, ma in realtà dal grande predecessore lo divide più di quanto non lo unisca. Per cominciare dalla convinzione che l'elemento considerato dall'altro più debole, l'elemento romanzesco, l'invenzione, sarebbe presto e definitivamente sconfitto dal "vero storico", Vassalli prende notevoli distanze dal maestro. Fin da Mareblù, attraverso La Chimera, e poi nell'ultima raccolta di racconti, egli mette a nudo quel che l'altro si è limitato solo a suggerire ${ }^{3}$ :

È l'odio puro: astratto, disincarnato, disinteressato; quello che muove l'universo, e che sopravvive a tutto. L'amore umano, tanto cantato dai poeti, a confronto dell'odio è quasi un fatto inesistente: un granello d'oro nel grande fiume della vita, una perla nel mare del nulla e niente più (La Chimera, pp. 73-74).

L'odio è l'ultimo dei tabù: qualcosa di cui si può parlare soltanto con infinite cautele, e che non si presenta mai con il suo vero nome, ma sempre mascherato di ragioni ipocrite. (Vassalli, Improvvisi)

Le differenze più vistose riguardano il piano dell'idea. Vassalli è uno scettico. Il suo scetticismo non lascia spazio alla Provvidenza e a Dio cristiani. È scettico al punto di sembrare un erede di Leopardi anziché di Manzoni. Alla fede infrangibile nella divina Provvidenza di quest'ultimo oppone un atteggiamento agnostico e un'immagine del mondo disincantata. Con fervore si cimenta sulle posizioni ostili alla superstizione diffusa dalla Chiesa, oggi come nel passato, accusandola di quel che con George Steiner si potrebbe definire la "paganizzazione" dell'eredità monoteistica ${ }^{4}$. Ma pare che il primo maestro di Vassalli sia Friedrich Nietzsche. Vassalli non crede in nulla, o meglio, crede nel Nulla di cui fa il fulcro di

"ideologicamente" quello di sempre, che vede la storia umana come un luogo di violenza e di bestialità, con la differenza che oggi entrambe (cfr. G. Bertone, "Ciao alla modernità") si sono normalizzate.

2. Scrive Cristina Nesi: "Non possiamo escludere che, a determinarne l'esigenza [di scrivere sul futuro], sia stato proprio un irriverente bisogno di sbeffeggiare chi gli aveva 'contristato l'anima per anni con l'etichetta di romanziere storico'. Adesso, cari critici, vedetevela un po' con il futuro! Si sarà detto lo scrittore." (Nesi, 2005, p. 90).

3. L'autore resta dell'avviso che Manzoni avrebbe detto esattamente le stesse cose sul carattere degli Italiani, se dalla modernità non lo separasse il Risorgimento: Manzoni vedeva i difetti, ma si adoperò a dissimularli, in vista della nazione italiana che ancora doveva nascere. Sostiene Giorgio Ficara che il male è in Vassalli un sogno perverso dell'umanità, e la pazzia del sangue versato rappresenti il punto culminante della storia.

4. La Chiesa cattolica, per rendere il patrimonio di Abramo, Isacco e Giacobbe più sopportabile agli umani lontani dalla dirittura di santi e di martiri, avrebbe introdotto indulgenze, reliquie, componende. La Chimera 
una nuova religione. Il nichilismo concepito come inerzia vitale diventa per lo scrittore l'essenza della materia e sostanza del mondo. La vita, nei romanzi vassalliani, consiste di un movimento sterile, mortale, ed è una malattia della materia; la morte ripristina l'equilibrio della materia, ripeterà ancora in $3012 \mathrm{e}$ in Stella avvelenata. Lo capisce perfino Antonia, protagonista de La Chimera:

Le sembrava di capire, finalmente!, qualcosa della vita: un'energia insensata, una mostruosa malattia che scuote il mondo e la sostanza stessa di cui sono fatte le cose. [...] la giustizia, la legge, Dio, l'Inferno - sono storie più fragili d'un sogno. ( $\mathrm{La}$ Chimera, p. 292)

Il nichilismo di marca vassalliana non è di carattere nirvanico, autosufficiente, contemplativo, bensì rimarca l'atteggiamento disincantato e disilluso di chi prova tuttavia un autentico interesse, preoccupazione e impegno per le cose di questo mondo e di questa società di cui si lamentano i maggiori vizi composti in un atto di accusa quale possono essere considerati sia Il neoitaliano che Gli Italiani sono gli altri. Infine si dissocia dai "risentiti", per dirlo con Nietzsche, e salva un atteggiamento eroico di chi sa guardare il mondo in faccia e continua ad andare avanti senza facili illusioni, senza la "menzogna religiosa", senza le utopie. Il Nulla è la legge che governa tutto in un mondo in cui non c'è posto per l'amore, per l'amicizia, per l'altruismo, e dove tutto cade presto in oblio. Il vizio capitale che lo scrittore lamenta nei suoi connazionali (il Casanova di Dux di Vassalli è un prototipico italiano: un fanfarone servile, l'opportunistico conservatore, l'egocentrico Narciso) è quello di amare e di sapere facilmente dimenticare, come hanno saputo dimenticare il proprio passato (ne parla in L'oro del mondo e ne Il Cigno, cfr. Nesi, p. 85).

Smarrita la fede in un Dio cristiano e nella manzoniana Provvidenza, Vassalli con veemenza professa la sua fede nel Nulla, così da farci pensare che egli avverta di quel Dio (o di un dio) una dolorosa mancanza, e che cerchi altre forme di spiritualità e di trascendenza, che provi la stessa nostalgia del sacro smarrito presente in Nietzsche che implora l'ignoto suo Dio, da cui si sente abbandonato, di tornare a lui. Vassalli soffre la mancanza di quel Dio inesperibile perché non rintracciabile nelle cose di questo mondo, e di cui non resta che il vuoto. Si dà a una spiritualità laica - per dirlo con un ossimoro - per cui la salvezza, che non può essere garantita da una divinità, è derivabile dall'unica forma di sopravvivenza

presenta l'esempio vistoso di una simile "paganizzazione" da una parte, e di emarginazione o, in casi estremi (come dimostra la caccia alla strega Antonia), di eliminazione di chi non vi si adegua. 
che, secondo Vassalli, ci è data: nella memoria degli altri a cui viene tramandata la nostra storia, se essa merita di essere salvaguardata. Chi non crede alla vita eterna (e lo scrittore sottolinea a più riprese di non credere in nulla) ritiene importante fare in modo che dopo la morte fisica un uomo abbia a disposizione la sopravvivenza costituita dalla propria storias. Per questo il mestiere dello scrittore, Vassalli ne è convinto, ha a che fare col sacro. Il primo vero protagonista dei suoi innumerevoli romanzi è lo stesso scrittore, il suo mestiere e la letteratura che accoglie questi racconti. $\mathrm{Ne}$ troviamo le estreme riprove ancora in Stella avvelenata che racconta una spedizione verso la terra mitica di Atlantide, il cui protagonista, il capitano Cat, incarna la necessità umana di raccontarsi, di sapersi raccontati. Paradossalmente per uno scrittore che si dice ateo, ne consegue che il mestiere dello scrittore abbia a che fare con una forma di trascendenza. Vassalli, infatti, recupera dal nulla e salva dalla dimenticanza le storie di singoli individui ${ }^{6}$. Senza l'intervento dello scrittore, del raccontatore, della nostra vita non resta che terra, cenere, ombra, nulla. Soltanto qualche volta di un uomo resta la sua storia, a patto che vivendo abbiamo tenacemente teso a qualcosa, se siamo rimasti fedeli fino in fondo a un miraggio, una Chimera, se abbiamo saputo inseguire un desiderio senza cedere quando il nostro percorso è stato ostacolato dall'ostile comunità, dal costume, dal potere, dall'opinione pubblica.

Diciamo per inciso che risulta pertinente a proposito il lungo apprendistato vassalliano tra le file dei neoavanguardisti, la cui poetica esaltava la fede nella parola e la patologia della lingua (la sintassi inesistente, frasi fatte di elenchi di nomi propri, citazioni latine, litanie di parole sconnesse) alludeva alla patologia del mondo reale. In quegli anni scrive Narcisso. Ed è lì, in quell'esperienza, che si forma la sua fede, quasi religiosa, nel potere della parola (del verbo!) che le è devoluto in via esclusiva. Il potere di creare, di chiamare a esistere, di salvare dal non essere, di tramandare. La parola rimane per Vassalli (tranne la breve parentesi testimoniata da

5. La notte della cometa, romanzo-verità (dopo cui lo scrittore rassegna le armi, disgustato da una massa di film melensi, biografie deliranti, strizzatine d'occhi, premi letterari a Campana intitolati, alla compagnia di villeggianti che ogni estate si riunisce a Marradi per assegnarli, constata il 26/II/2003 sul Corriere della Sera: "Hanno vinto loro. Addio, Dino". In Nesi, 2005, p. 60) come lo definisce l'autore stesso, è pervaso dall'insistente assillo della perdita della memoria. Ogni ricordo si perde nel volgere al massimo di qualche decennio.

6. Per Vassalli la storia non esiste, esistono soltanto - come ripeterà in Un infinito numero - le storie umane dentro il passato. Lo scrittore preferisce quel che si definisce a volte la visione "creaturale" della storia - propria delle vittime della storia ufficiale; ovvero dei singoli individui nel loro agire nella storia. Gli interessano le storie umane, perché la storia umana è un infinito numero di storie che finiscono per contraddire l'idea stessa della Storia univoca. 
L'arrivo della lozione ${ }^{7}$ ) l'unica ancora di salvezza, in grado di opporsi al nulla del reale in cui si dissolvono senza lasciare traccia alcuna le storie umane. L'autore scrive che le parole costituenti l'antidoto al mondo in disordine (quel disordine comprendeva anche le istituzioni letterarie di fronte a cui Vassalli era sempre stato molto critico, le politiche editoriali dei critici e dei recensori, le scelte e i calcoli delle giurie dei premi letterari ecc.) riescono da sole a far fronte al mondo "privo di forza e sostanza inconoscibile innominabile nullo" (Il millennio che muore, p. 8$)^{8}$.

Abbiamo detto che lo scrittore, nell'espletare il suo "sacro" mestiere, abbina alla riflessione sul carattere degli italiani ${ }^{9}$ la sua ricerca sui fatti del passato di cui abbiamo solo una conoscenza parziale, imperfetta. Al dubbio storico o storiografico si affianca lo scetticismo civile, morale, ideologico che gli fa vedere la secolare attività umana in termini di sperpero, un inutile quando non meramente distruttivo dibattersi di milioni di persone e di singoli individui. Vassalli si assume il compito dell'artista (e dell'intellettuale) che consiste nel riempire gli interstizi della storia, e poi nel ricordare, tramandare la memoria, nel salvarla dalla dimenticanza. Capiamo allora come l'interesse per il passato possa essere alimentato, e alimentare a sua volta, la passione, che pare una passione civile quanto morale.

Lo scrittore sente la nostalgia della divinità inesistente, nella stessa misura del suo maestro Nietzsche il cui problema centrale sono le conseguenze per l'uomo contemporaneo della morte di Dio. Dopo che è morto Dio, per Nietzsche è rimasta la moralità, la moralità dei deboli e quella degli spiriti forti, la Herrensmoral. I deboli, malati, vili, sconfitti, risentiti contro la vita, non sanno reagire adeguatamente agli stimoli della vita, alla vitalità spontanea e aggressiva, vivono di illusione (il concetto di "maschera"). Nel loro "risentimento" Nietzsche individua la radice della morale cristiana (che egli assimila alla "morale degli schiavi"). I deboli, della propria debolezza e viltà fanno una virtù. Invece la morale dello

7. Vassalli accusa nel romanzo scritto nel 1976 lo scialo delle parole, vuote e nate deliberatamente per ingannare, che avrebbero trascinato molti verso la rivoluzione che doveva essere una lozione magica (a cui allude il titolo L'arrivo della lozione: rivo-luzione), soffermandosi sull'uso improprio, indebito di alcune parole come pace, giustizia, democrazia, sinistra, svuotate del loro significato e piegate verso altri scopi.

8. Stefano Tani vede nell'esperienza vassalliana di Narcisso e di Tempo di màssacro "una nevrotica autoreferenza" per cui il linguaggio raffigura non meno la letteratura di allora in stato cadaverico, del mondo in decomposizione, l'uomo in stato demente, esprimendo "una situazione umana di apocalittica claustrofobia" (Tani, I990, p. 5I).

9. L'ispirazione, ricordiamo, gli è venuta dal suo amico, editore, Giulio Bollati, che lamentava l'abitudine al trasformismo, o al camaleontismo politico e l'impotenza degli intellettuali italiani di fronte a un neodarwinismo sociale allo stato grezzo e violento. 
spirito forte richiede di porsi indipendentemente dalla moralità di risentimento, dimostrare di essere in pieno possesso di sé, sapendo e sopportando serenamente la "menzogna religiosa". Diversamente dalla morale dei risentiti, questa non offre regole, ma consiste in autoespressione e porta a trovare la verità in se stessi. Pochi saranno coloro cui sarà consentito risalire dalla caverna e vedere il sole, per così dire. A fronte di tale divisione Nietzsche dello Zarathustra lasciava un margine all'iniziativa umana. Vassalli sembra credere nel libero arbitrio, nella forza dello spirito che permette all'uomo di elevarsi, di vincere il potente determinismo per cui siamo quello che siamo, di conseguenza a quello che abbiamo fatto una volta per sempre. In più, lo scrittore non cerca di capire o di venire a patti con il meccanismo che sta dietro quel determinismo, non lo problematizza, lo constata e basta. Il mondo va dove esso vuole, non dove vorremmo che andasse, ripete in La Chimera, in Un infinito numero e in L'archeologia del presente. Se la morale dei forti è il principio della vitalità, la storia dell'Occidente, secondo Nietzsche e per Vassalli, non è altro che la storia della negazione della vita, la storia dei vili.

$\mathrm{Ne}$ La Chimera vi sono almeno due spiriti forti che sanno andare contro e, nonostante tutto, lungo la loro strada. Il primo è il vescovo di Novara, un grande moralista, di rara forza d'animo, Carlo Bascapè, un riformistaintegralista, appartenente alla fazione dei nemici del clero "ipocrita e stagnante di quella Roma, e di quei Papi, e di quegli anni” (La Chimera, p. 220). Il papa proprio per queste sue doti ha relegato questo ostacolo vivente alla rilassatezza dei costumi romani più lontano possibile dalla sede centrale. Fallitone il disegno di ascesi e severa disciplina dei fedeli, a cui imponeva messe, preghiere, castighi e digiuni, Bascapè, pur fedele alla sua chimera, fallisce nel proposito di riformare la Chiesa che continua a essere come "la Babilonia delle antiche scritture: un monumento alle cose del mondo e alla politica del mondo" (ibidem, p. 26I) ${ }^{\text {Io }}$. La Chiesa rimane quella che è, quella che fu, "paganizzata", come spesso la rappresenta lo scrittore anticlericale, popolata da preti (alla don Teresio che si fa portare decime, regalìe, devozioni e elemosine per "scongiurare la presenza dia-

Io. Sembra pertanto non casuale che quando, durante il pontificato di Clemente VIII e poi Paolo VI, si sviluppa l'Inquisizione, al vescovo novarese cominciano ad accadere cose strane. Non a caso egli prima viene coinvolto e accusato di aver acquistato false reliquie, poi come su richiesta sopraggiunge "la causa giusta al momento giusto, mandata dalla divina Provvidenza e irrinunciabile” (La Chimera, p. 225). L'inquisitore Manini, nemico del vescovo, non poteva immaginare un'occasione migliore per distruggere lo scomodo vescovo, per allontanarlo per sempre dalla politica: un caso di eresia e di patto col diavolo nella diocesi di Bascapè! Non si poteva sperare in meglio! Il Tribunale del Sant'Uffizio di Novara ha tutto l'interesse a portare a termine il processo della "strega di Zardino" per rivendicare la propria autonomia nei confronti di Bascapè e della Chiesa di Roma. 
bolica" nei boschi circostanti il villaggio), da preti-avvocati, preti-usurai, preti-proprietari di bordelli, venditori di indulgenze, trafficatori di false reliquie che in seguito venivano autenticate per denaro dal monsignore di modo che tutta la catena alimentare (dal tombarolo, al notaio, al cardinale prefetto delle Catacombe) fosse soddisfatta. Tutto ciò fiorisce e prospera perché Dio vero non esiste, o si è ritirato dalla cose umane. Per secoli la fede è servita ad arrecare violenza, torturare e uccidere in nome di Dio. Antonia, la seconda figura forte nel romanzo vassalliano, che fin da piccola spicca per grazia e bellezza, ma innanzi tutto per intelletto (si interroga sul senso della vita umana, al di là delle storie insensate raccontate dalle suore nell'orfanotrofio, e in seguito, già a Zardino, ha il coraggio di dire in pubblico che inferno e paradiso non esistono se non quaggiù in terra, che oltre alla morte c'è "un gnente grande come il cielo; et in quel gnente le favole de preti” [ibidem, p. I93]), sarà sconfitta dall'odio, intolleranza, interessi particolari, cupidigia dei preti, lotte tra funzionari della Chiesa e dalla gratuita violenza della gente vile, di quei deboli che hanno bisogno di una giustificazione superiore per le proprie azioni. Ne rimane tuttavia qualcosa, un esempio di tenacia e inflessibilità di chi ha difeso la sua chimera a costo di morte. Grazie alla parola, ne rimane la storia.

Per Vassalli la morte di Dio e la clamorosa sfiducia nel mandato della Chiesa come istituzione comportano la necessità di ripensare e riproporre il proprio compito di scrittore (artista e intellettuale). I filosofi, constatata la morte di Dio, e che si sentono, afferma Nietzsche, come illuminati dai raggi della nuova aurora, spiccano il volo, sciolgono le vele alle loro navi, muovendo incontro al rischio di nuova conoscenza e di un nuovo aperto mare. Uno scrittore potrebbe cercare di imitare il maestro del nichilismo nell'avere il coraggio di andare senza Dio, acquistando la nuova leggerezza, rovesciando le vecchie tavole senza temere le regole e le leggi. Morti tutti gli dèi, evviva il superuomo? Sì, se pensiamo che soltanto uno spirito forte può scartare tutte le utopie allo stesso tempo, dissipare tutte le illusioni perché esse non possono metterci al riparo della sofferenza, guardare dritto in faccia il destino umano, i vizi della società e di individui singoli ${ }^{\text {II }}$, l'insensatezza della vita stessa governata dall'inerzia della materia, del mondo caotico e materialistico, della storia il cui motore è l'odio.

II. Le utopie crollano perché partono dall'idea sbagliata che l'uomo sia buono e perfettibile, mentre esso è malvagio, incorreggibile, peccatore. "Chi ha scommesso sull'uomo buono, chi ha pensato che le vicende umane si potessero razionalizzare e perfezionare, ha sempre perso in tempi brevi. Basta pensare al marxismo. Invece dura da due mila anni la parabola della Chiesa che parte dal peccato originale, dall'uomo malvagio." (Nesi, 2005, p. 99, nota 266) 
Contro l'eterno ritorno di quell'odio che nullifica tutto non vale nessun sacrificio (quello originario di Cristo o quello ritualizzato e imitato dopo, come nel gesto di Mattio che si autocrocifigge, in Marco e Mattio), il che rende insensata e inutile ogni azione umana (La Chimera). È un lungo percorso, dai tempi del romanzo Abitare il vento (1980) in cui Vassalli ci convinceva che pensare di vivere fuori dal tempo, di abitare il vento è illusorio, come è vano sperare di liberarsi da tutto, distruggere, abolire quel che ci sta intorno. Il tempo e le ideologie non si possono rinnegare, anzi si finisce sempre per esservi invischiati, per cui vivere il vento è una metafora della libertà impossibile, inaccessibile. Da ultimo, nella sua raccolta $L a$ morte di Marx e altri racconti, in cui lo scrittore giunge a rinnegare alla maniera postmoderna le grandi narrazioni, Vassalli si congeda dai maestri della modernità: Kafka, Marx (il dottor Marx qui ha idee diverse dal suo omonimo teorico del proletariato e della lotta di classe, ed è convinto che la parte razionale dell'uomo è debole rispetto a quella irrazionale e che le ideologie e le utopie non sono servite a nulla, e noi impiegheremo ancora chissà quanti millenni per diventare davvero civili), e anche Freud (le storie qui raccontate sono tutte di amore mancato, impossibile). Allo stesso tempo lo scrittore pone, modernisticamente, se stesso, scrittore e artista - quasi un sacerdote - al posto di Dio, in maniera eroica, dopo aver vissuto e scontato l'intera parabola che lo ha portato dal mettersi in gioco ne L'oro del mondo (perché, come usava sottolineare, egli ha voluto guardare anche la pagliuzza nel proprio occhio mentre scorgeva la trave in quello degli altri), attraverso l'adesione alle grandi utopie del secolo con il conseguente tentativo di staccarsi da tutto e di "abitare il vento", fino all'eroica resa delle illusioni tra cui una delle più grandi era quella di cambiare il mondo (come il vescovo Bascapè de La Chimera, come Leo e Michela di L'archeologia del presente, idioti, coloro che hanno speso le loro vite per far diventare il mondo perfetto, ma che a prezzo di enormi fatiche sono riusciti a portarlo soltanto sull'orlo del baratro).

Lo scrittore insegue sempre nuove chimere per descriverle, perché "sono queste illusioni che aiutano l'umanità a vivere” (Nesi, p. 79), egli solo è capace di perdurare tra le rovine delle ideologie, narrazioni e storie crollate, nel nostro oggi destrutturato, deideologizzato, disingannato. Cerca, coglie e descrive momenti di vita di coloro che nella storia hanno seminato illusioni per pochissimo tempo, prima di rimanerne vittime, di venirne travolti poco dopo, in un mondo dei cui limiti Vassalli è progressivamente più cosciente. Il mondo materiale (e la fede nei dischetti bianchi dell'Aspirina, di cui si dichiara fanatico il narratore di Archeologia del presente, p. I06) prevale sulla vitalità e sull'idealità dei più, e ciò diventa strumen- 
tale nel percorso vassalliano verso la sospirata leggerezza che Nietzsche riservava (in premio?) agli spiriti forti.

A volte, specialmente d'estate, capita anche a me, come capitava a don Marco e Mattio Lovat, di alzare gli occhi verso il cielo stellato. E mi piace perdermi col pensiero in quel pulviscolo di sistemi solari che si vedono tra una costellazione e l'altra, $\mathrm{e}$ in quel buio che c'è dietro i sistemi solari, dove si muovono inutilmente milioni di mondi. Soffermarmi a riflettere sull'infinità di quello spèrpero che chiamiamo universo mi fa bene e mi aiuta a star bene. Che altro sono le nostre impercettibili vite e le nostre microscopiche storie, se non sperpero nello sperpero? (Marco e Mattio, p. 5; la sottolineatura è mia)

\section{Bibliografia}

Bertone G., "Ciao alla modernità", L'Indice, n. 5, 2006, p. I2.

Bollati G., L'italiano: Il carattere nazionale come storia e invenzione, Torino, Einaudi, 1983.

Di Stefano P., "Vassalli: 'I delitti del pio Enea", Il Corriere della Sera, I4 settembre 1999 .

Ficara G., "Seicento al rogo", Panorama, 23 gennaio I990.

Nesi C., Sebastiano Vassalli, Fiesole, Cadmo, 2005.

Riccardi C., "Intervista a Sebastiano Vassalli", Autografo, n.25, I992, pp. 7I-84, qui p. 75 .

Tani S., Il romanzo di ritorno, Milano, Mursia, I990.

Vassalli S., 30I2: L'anno del profeta, Torino, Einaudi, 1995.

—, Abitare il vento, Torino, Einaudi, 1980.

—, Archeologia del presente, Torino, Einaudi, $200 \mathrm{I}$.

-, Dux: Casanova in Boemia, Torino, Einaudi, 2002.

-, Gli Italiani sono gli altri. Viaggio (in undici tappe) all'interno del carattere nazionale italiano, Milano, Baldini Castoldi Dalai, 2003.

—, Il Cigno, Torino, Einaudi, 1993.

—, Il millennio che muore, Torino, Einaudi, 1972.

-, Il neoitaliano. Le parole degli anni Ottanta, Bologna, Zanichelli, I989.

—, "Improvvisi: le nuove frontiere dell'eterno odio", Il Corriere della Sera, 27 aprile 1998.

—, La Chimera, Torino, Einaudi, I990.

—, L'arrivo della lozione, Torino, Einaudi, 1976.

—, L'oro del mondo, Torino, Einaudi, 1987.

—, La morte di Marx e altri racconti, Torino, Einaudi, 2006. 
—, La notte della cometa: Il romanzo di Dino Campana, Torino, Einaudi, I984.

—, Marco e Mattio, Torino, Einaudi, 1992.

—, Mareblì, Torino, Einaudi, 1982.

—, Narcisso, Torino, Einaudi, 1968.

-, Stella avvelenata, Torino, Einaudi, 2003.

—, Tempo di màssacro, Torino, Einaudi, I970.

-, Un infinito numero: Virgilio e Mecenate nel paese dei Rasna, Torino, Einaudi, 1999. 